\title{
CANARD CYCLES AND HOMOCLINIC BIFURCATION IN A 3 PARAMETER FAMILY OF VECTOR FIELDS ON THE PLANE
}

\author{
PAUlo Ricardo DA Silva
}

Abstract

Let the 3-parameter family of vector fields given by

$$
y \frac{\partial}{\partial x}+\left[x^{2}+\mu+y\left(\nu_{0}+\nu_{1} x+x^{3}\right)\right] \frac{\partial}{\partial y}
$$

with $\left(x, y, \mu, \nu_{0}, \nu_{1}\right) \in R^{2} \times R^{3}$ ([DRS1]). We prove that if $\mu \rightarrow$ $-\infty$ then (A) is $C^{0}$-equivalent to

(B) $\quad\left[y-\left(b x+c x^{2}-4 x^{3}+x^{4}\right)\right] \frac{\partial}{\partial x}+\varepsilon\left(x^{2}-2 x\right) \frac{\partial}{\partial y}$

for $\varepsilon \downarrow 0, b, c \in R$. We prove that there exists a Hopf bifurcation of codimension 1 when $b=0$ and also that, if $b=0, c=12$ and $\varepsilon>0$ then there exists a Hopf bifurcation of codimension 2. We study the "Canard Phenomenon" and the homoclinic bifurcation in the family (B). We show that when $\varepsilon \downarrow 0, b=0$ and $c=12$ the attracting limit cycle, which appears in a Hopf bifurcation of codimension 2, stays with "small size" and changes to a "big size" very quickly, in a sense made precise here.

\section{Introduction}

Let $\chi$ the set of $C^{\infty}$ vector fields on the plane and $\chi_{0} \subset \chi$, the set of vector fields with a singularity at $(0,0)$.

(1) $\chi_{0}=\left\{P(x, y) \frac{\partial}{\partial x}+Q(x, y) \frac{\partial}{\partial y} \mid P, Q \in C^{\infty}, P(0,0)=Q(0,0)=0\right\}$.

Consider the equivalences introduced by the following definitions.

\section{Definition 1.1.}

a) $X, Y \in \chi$ are called $C^{0}$-equivalent if there exists a homeomorphism $h: R^{2} \rightarrow R^{2}$ sending $X$-orbits to $Y$-orbits in a sense preserving way.

b) $X, Y \in \chi_{0}$ are called $C^{\infty}$-equivalent if there exists a diffeomorphism $g: R^{2} \rightarrow R^{2}$, fixing $(0,0) \in R^{2}$, such that $Y(p)=$ $\left[g^{\prime}(p)\right]^{-1} \cdot X(p), p \in R^{2}$. 


\section{Definition 1.2.}

a) A $k$-parameter family of vector fields on $R^{2}, X_{\lambda}$, with $\lambda \in R^{k}$ denoting the parameter, is defined to be a vector field $a(m, \lambda) \frac{\partial}{\partial x}+$ $b(m, \lambda) \frac{\partial}{\partial y}, m=(x, y) \in R^{2}$, where the coefficient functions $a$ and $b$ are $C^{\infty}$ with respect to $(m, \lambda) \in R^{2} \times R^{k}$. We say that $X_{\lambda}$ is a $k$-parameter unfolding of $X_{0}$.

b) $X_{\lambda}$ and $Y_{\mu}, \mu, \lambda \in R^{k}$, are (fibre) $C^{0}$-equivalent if there exist homeomorphisms $\mu=\phi(\lambda)$ and $h_{\lambda}: R^{2} \rightarrow R^{2}$ such that $h_{\lambda}$ is a topological equivalence between $X_{\lambda}$ and $Y_{\phi(\lambda)}$.

Let $X_{0} \in \chi_{0}$. Consider the Jordan form $J X_{0}$ of $\left[\begin{array}{cc}\frac{\partial P}{\partial x} & \frac{\partial P}{\partial y} \\ \frac{\partial Q}{\partial x} & \frac{\partial Q}{\partial y}\end{array}\right]$ at $(0,0) \in R^{2}$ $([\mathbf{M}])$.

(2)

$$
\begin{aligned}
& T_{11}:\left[\begin{array}{ll}
a & b \\
0 & c
\end{array}\right] \quad a \neq 0, c \neq 0 \text { and } b=0 \text { or } a=c \neq 0 \text { and } b=1 \\
& T_{12}:\left[\begin{array}{cc}
a & b \\
-b & a
\end{array}\right] \quad a \neq 0, b \neq 0 \\
& T_{2}:\left[\begin{array}{cc}
a & 0 \\
0 & 0
\end{array}\right] \quad a \neq 0 \\
& T_{3}:\left[\begin{array}{cc}
0 & -a \\
a & 0
\end{array}\right] \quad a \neq 0 \\
& T_{4}:\left[\begin{array}{cc}
0 & 1 \\
0 & 0
\end{array}\right] \\
& T_{5}:\left[\begin{array}{ll}
0 & 0 \\
0 & 0
\end{array}\right] .
\end{aligned}
$$

Let $W_{i}=\left\{X_{0} \in \chi_{0} \mid J X_{0} \in T_{i}\right\}$ where $T_{1}=T_{11} \cup T_{12}$. Thus $\chi_{0}=W_{1} \cup W_{2} \cup W_{3} \cup W_{4} \cup W_{5}$. $W_{1}$ is the set of vector fields with hyperbolic singularity at $(0,0)$. One obtains for generic $k$-parameter families (with any $k$ ) the so called generalized saddle-node bifurcation of codimension $k$ which is an unfolding of $X_{0} \in W_{2}$ but whose restriction to center manifold starts with non-zero terms of order $k$. For generic $k$-parameter families $(k \geq 2)$ one finds the generalized Hopf bifurcation where such Hopf bifurcation of codimension $k$ is a $k$-parameter unfolding of $X_{0} \in W_{3}$ and whose radial component of the normal form in polar coordinates starts with non-zero terms of order $2 k+1$.

The study of the unfoldings of $X_{0} \in W_{4}$ starts with the BogdanovTakens bifurcation $([\mathbf{R W}])$. If $X_{0} \in W_{4}$ then, according [DRS1], $j^{2} X_{0}$ 
is $C^{\infty}$-equivalent to

$$
y \frac{\partial}{\partial x}+\left(a x^{2}+b x y\right) \frac{\partial}{\partial y} .
$$

If $a \neq 0$ we say that $X_{0} \in W_{4}$ has singularity of the kind cusp. For the case $a=0$, see [DRS2].

Generically $a b \neq 0$ and $j^{2} X_{0}$ is $C^{\infty}$-equivalent to $([\mathbf{D}])$

$$
y \frac{\partial}{\partial x}+\left(x^{2} \pm x y\right) \frac{\partial}{\partial y}
$$

Bogdanov and Takens showed that, generically, any local 2 parameter unfolding of (4) is fibre $C^{0}$-equivalent to

$$
X_{\mu, \nu}=y \frac{\partial}{\partial x}+\left[x^{2}+\mu+y(\nu \pm x)\right] \frac{\partial}{\partial y} .
$$

Dumortier, Roussarie and Sotomayor ([DRS1]) studied $X_{0} \in W_{4}$ with $j^{2} X_{0} C^{\infty}$-equivalent to

$$
y \frac{\partial}{\partial x}+a x^{2} \frac{\partial}{\partial y} .
$$

They showed that, generically, $j^{4} X_{0}$ is $C^{\infty}$-equivalent to

$$
y \frac{\partial}{\partial x}+\left(x^{2} \pm x^{3} y\right) \frac{\partial}{\partial y} .
$$

Generically, any local 3 parameter unfolding of (7) is fibre $C^{0}$-equivalent to

(8) $X_{\mu, \nu_{0}, \nu_{1}}=P \frac{\partial}{\partial x}+Q \frac{\partial}{\partial y}=y \frac{\partial}{\partial x}+\left[x^{2}+\mu+y\left(\nu_{0}+\nu_{1} x \pm x^{3}\right)\right] \frac{\partial}{\partial y}$.

The bifurcation set of $X_{\mu, \nu_{0}, \nu_{1}}$ is the smallest closed subset $\sum \subset$ $R^{3}\left(\mu, \nu_{0}, \nu_{1}\right)$ such that the topological type of $X_{\mu, \nu_{0}, \nu_{1}}$ for $\left(\mu, \nu_{0}, \nu_{1}\right) \in$ $R^{3}\left(\mu, \nu_{0}, \nu_{1}\right) \backslash \sum$ is locally constant. In Figure 1 we have the bifurcation set of $\left(8^{+}\right)$. It is composed of 9 surfaces and 5 curves.

Figure 1 shows the intersection of the bifurcation set with the sphere $S_{\delta}=\left\{\left(\mu, \nu_{0}, \nu_{1}\right) \mid \mu^{2}+\nu_{0}^{2}+\nu_{1}^{2}=\delta^{2}, \mu<0\right\}$, with $\delta>0$ small. The parameter values for which $\left(\frac{\partial P}{\partial x}+\frac{\partial Q}{\partial y}\right)(-\sqrt{-\mu}, 0)=0$ give the surface $H$ (Hopf bifurcation). $H$ is composed of two parts separated by the 
curve $\mathrm{H}_{2}$ (Hopf bifurcation of codimension 2). In one of them, when the parameter value crosses $H$, the focus changes the stability and we have the appearance of one repelling limit cycle. In the other part, the change of the stability gives the appearance of one attracting limit cycle. When the parameter value crosses $\mathrm{H}_{2}$ we have the appearance of two limit cycles, the inner one is attracting and the other is repelling. If $\left(\mu, \nu_{0}, \nu_{1}\right) \in L$ (homoclinic bifurcation) then $X_{\mu, \nu_{0}, \nu_{1}}$ has a homoclinic orbit. The parameter values on $L$ for which $\left(\frac{\partial P}{\partial x}+\frac{\partial Q}{\partial y}\right)(\sqrt{-\mu}, 0)=0$ give the curve $L_{2}$. $L$ is composed of two parts separated by the curve $L_{2}$ (homoclinic bifurcation of codimension 2). In one of them, the attracting limit cycles which appear along $H$ disappear along $L$. In the other part, the repelling limit cycles which appear along $H$ disappear along $L$. The cycles which appear along $H_{2}$ disappear along $L_{2}$. There exists a surface $C$ where the attracting limit cycle and the repelling limit cycle coalesce. If $\left(\mu, \nu_{0}, \nu_{1}\right) \in C$ then $X_{\mu, \nu_{0}, \nu_{1}}$ has a semistable cycle. For the parameter values on the region limited by $H, L$ and $C, X_{\mu, \nu_{0}, \nu_{1}}$ has two limit cycles. On $b_{1}(\delta)$ and $b_{2}(\delta)$ we have the Bogdanov-Takens bifurcations corresponding to the cases $\left(5^{+}\right)$and $\left(5^{-}\right)$. For the parameter values on $H \cap L$ we have simultaneous Hopf bifurcation and homoclinic bifurcation.
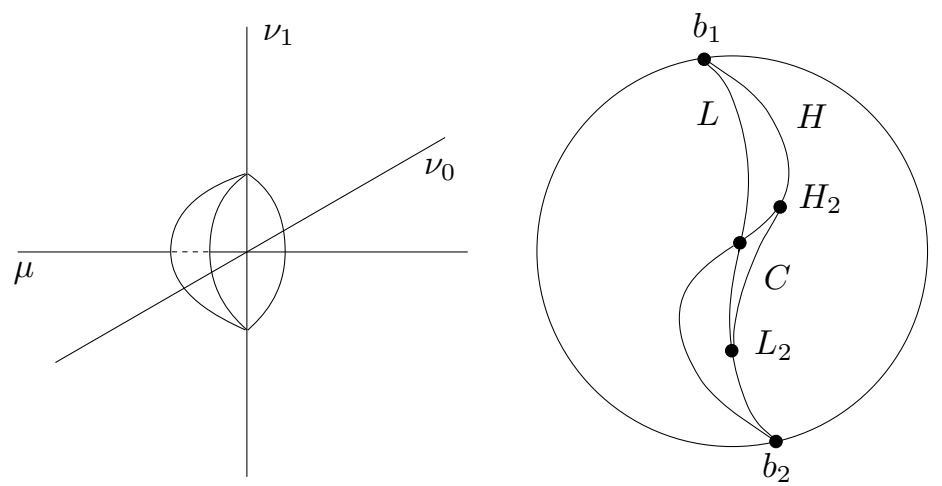

Figure 1. The Bifurcation Set on $S_{\delta}$.

In this work we study the surfaces $H$ and $L$ for $\mu \rightarrow-\infty$.

In section 2 we make some coordinate changes, such as the Liénard transformation, and prove that if $\mu \rightarrow-\infty$ then $\left(8^{+}\right)$is (fibre) $C^{0}$-equivalent to

$$
\left[y-F_{b, c}(x)\right] \frac{\partial}{\partial x}+\varepsilon\left(x^{2}-2 x\right) \frac{\partial}{\partial y}
$$

for $\varepsilon \downarrow 0, b, c \in R$. 
We denote $F_{b, c}$ the function given by

$$
F_{b, c}(x)=b x+c x^{2}-4 x^{3}+x^{4} .
$$

In section 2 we study the bifurcation set of the functions defined by (10). It is illustrated in Figure 2.

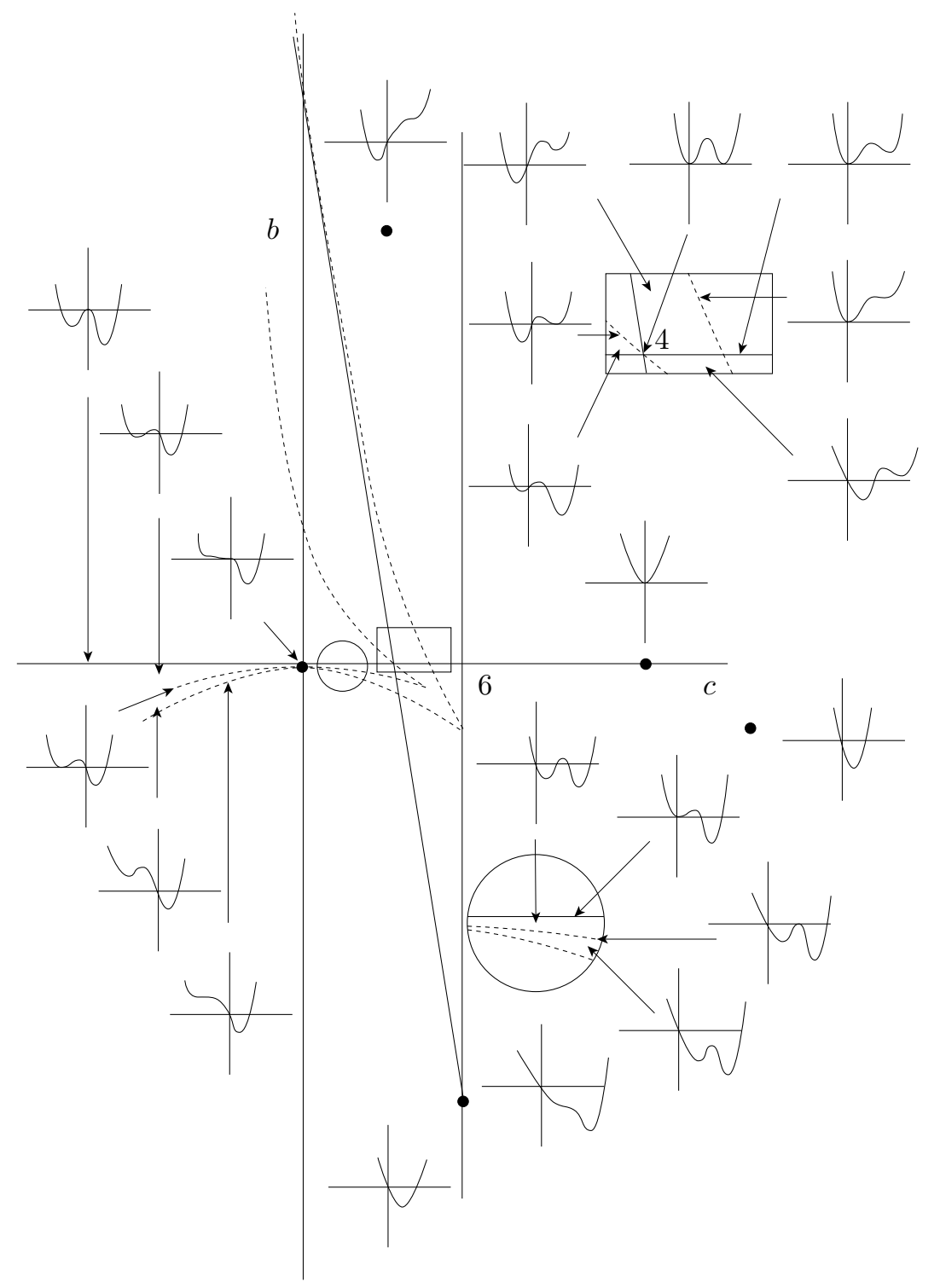

Figure 2. The Bifurcation Set of $F_{b, c}$. 
In section 3 we study the singularities of the family (9). If $\varepsilon=0$ then the singularities, with $(b, c)$ fixed, are on the curve

$$
L_{b, c}=\left\{(x, y) \mid y=F_{b, c}(x)\right\} .
$$

Except for the critical points, all the points on $L_{b, c}$ are normally hyperbolic singular points. The phase portrait is illustrated in Figure 3.
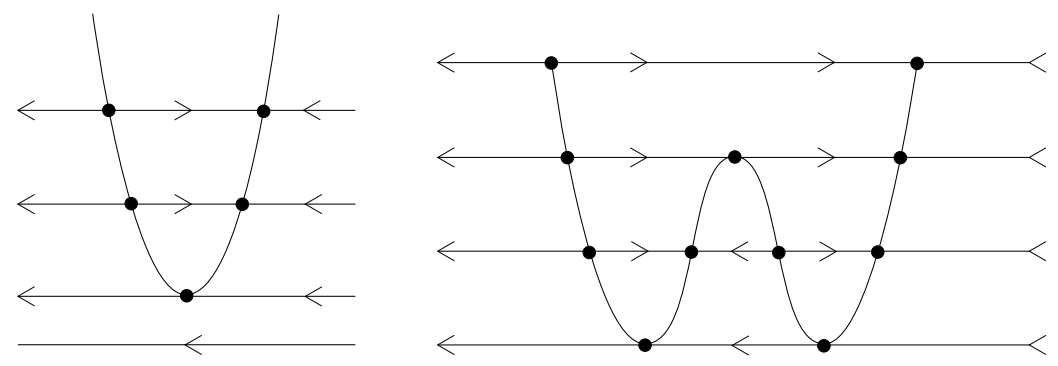

Figure 3. Phase Portrait of $(9)$ for $(\varepsilon, b, c)=(0,0,12)$

$$
\text { and for }(\varepsilon, b, c)=(0,0,4) \text {. }
$$

For $\varepsilon>0,(9)$ has two singularities: $(0,0)$ and $\left(2, F_{b, c}(2)\right)$.

$$
0<\varepsilon \leq b^{2} / 8 \quad \varepsilon>b^{2} / 8
$$

$$
\begin{array}{llll}
(0,0) & b>0 & \text { Attracting Node } & \text { Attracting Focus } \\
& b<0 & \text { Repelling Node } & \text { Repelling Focus }
\end{array}
$$

$\left(2, F_{b, c}(2)\right) \quad$ Saddle Saddle

Table I. Singularities of (9) for $\varepsilon>0$.

We have a special care to classify the singularity $(0,0)$ when $b=0$ because in this case the associated eigenvalues have real part equal zero. We prove in section 3 the existence of Hopf bifurcation on $H=\{b=0, c \neq 12\}$ and Hopf bifurcation of codimension 2 on $H_{2}=$ $\{b=0, c=12\}$. The cycles are so that inner one is repelling and the other is attracting. We study the behaviour of the cycles for $\varepsilon \downarrow 0$.

\section{Definition 1.3.}

a) Let $A, B \subset R^{2}$ compact sets. The Hausdorff distance between $A$ and $B$ is $d(A, B)=\max _{x \in A, y \in B}\{d(x, B), d(y, A)\}$.

b) $\Gamma_{b, c} \subset R^{2}$ is a "Limit Periodic Set" of $X_{\varepsilon, b, c}$ if there exist $\left(\varepsilon_{n}, b_{n}, c_{n}\right) \rightarrow(0, b, c)$ and $\Gamma_{n} \subset R^{2}$, limit cycle (or homoclinic orbit) of $X_{\varepsilon_{n}, b_{n}, c_{n}}$, such that $\Gamma_{n} \rightarrow \Gamma_{b, c}$, for the Hausdorff distance between the compact sets in $R^{2}$. 


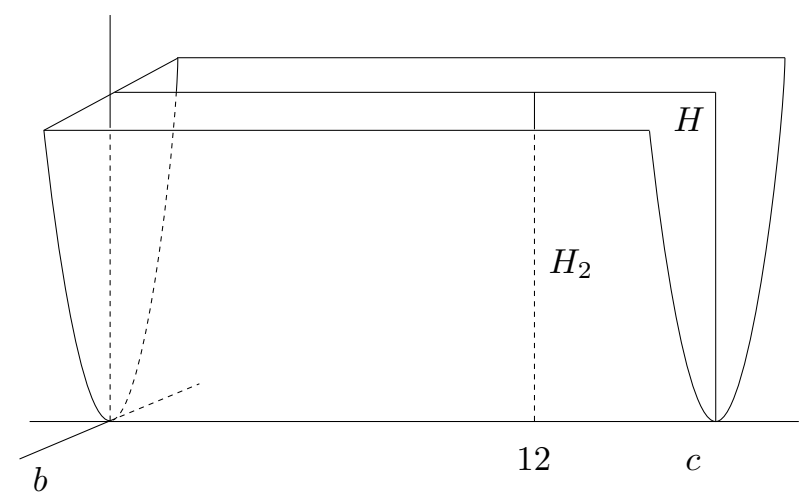

Figure 4. Hopf Bifurcation.

For $(\varepsilon, b, c) \rightarrow(0,0,12)$ the possible limit periodic sets are those for which $y$ is constant or $y=F_{b, c}(x)$. The limit periodic sets are known by "Canard Cycles". We use the term "canard" because the shape of the limit periodic sets of the Van der Pol's equation is like a duck $([\mathbf{E}])$.

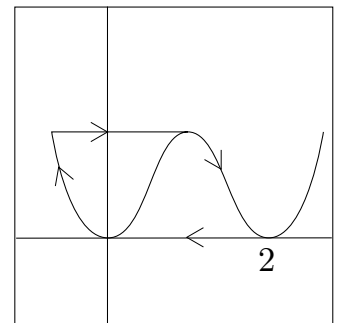

$\Gamma_{0,4}$

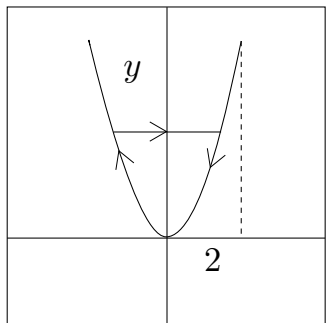

$\Gamma_{0,12}^{y}$

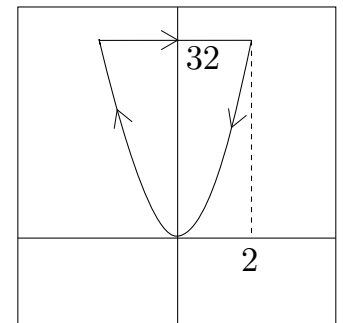

$\Gamma_{0,12}^{32}$

Figure 5. Limit Periodic Sets.

Using the method introduced in [DR1], which consists in global desingularization and center manifolds, we prove

Theorem 1.1. Let the family given by (9) and $\Gamma_{0,12}^{32}$ the compact set illustrated in Figure 5. There exist $\varepsilon_{0}>0, \delta>0$ and a surface $S=\left\{(\varepsilon, b, c) \mid b=\varphi(\sqrt{\varepsilon}, c), \varphi \in C^{\infty}, \varphi(0, c)=0,0 \leq \varepsilon<\varepsilon_{0}, 12-\delta<\right.$ $c<12+\delta\}$ such that if $(\varepsilon, b, c) \in S$ and $\varepsilon>0$ then two separatrices of the saddle point $\left(2, F_{b, c}(2)\right)$ give a homoclinic orbit which approaches $\Gamma_{0,12}^{32}$ for the Hausdorff distance.

With the same method one can find surfaces $S_{y}, 0 \leq y \leq 32$, such that if $(\varepsilon, b, c) \in S_{y}$ then the phase portrait of (9) has a limit cycle $\Gamma_{\varepsilon, b, c}^{y}$ and $\Gamma_{\varepsilon, b, c}^{y} \rightarrow \Gamma_{0,12}^{y}$, for the Hausdorff distance, for $(\varepsilon, b, c) \rightarrow(0,0,12)$. 
The divergence at $\left(2, F_{b, c}(2)\right)$ determines the stability of the homoclinic orbit. We have

$$
\operatorname{div} X_{\varepsilon, b, c}\left(2, F_{b, c}(2)\right)=0 \Leftrightarrow b=-4 c+16 .
$$

We say that the homoclinic orbit is non-generic if the divergence at $\left(2, F_{b, c}(2)\right)$ is zero.

Theorem 1.2. Let the family given by (9) and $\Gamma_{0,4}$ the compact set illustrated in Figure 5. There exist $\varepsilon_{0}>0, \delta>0$ and a surface $L=$ $\left\{(\varepsilon, b, c) \mid b=\psi(\sqrt{\varepsilon}, c), \psi \in C^{\infty}, \psi(0, c)=0,0 \leq \varepsilon<\varepsilon_{0}, 4-\delta<c<\right.$ $4+\delta\}$ such that if $(\varepsilon, b, c) \in L$ and $\varepsilon>0$ then two separatrices of the saddle point $\left(2, F_{b, c}(2)\right)$ give a homoclinic orbit $L_{\varepsilon, b, c}$ which approaches $\Gamma_{0,4}$. Besides the limit of the parameter values for which $X_{\varepsilon, b, c}$ has nongeneric homoclinic orbits for $\varepsilon \downarrow 0$ is $(0,0,4)$.

The surface $L$ is such that for $b<-4 c+16, L_{\varepsilon, b, c}$ is a repelling homoclinic orbit and for $b>-4 c+16, L_{\varepsilon, b, c}$ is an attracting homoclinic orbit.

According [ALGM], if $b=-4 c+16$ and $(\varepsilon, b, c) \in L$ then for any $\delta_{1}>0$ and $\delta_{2}>0$, there exists a perturbation $\widetilde{X_{\varepsilon, b, c}}$ of $X_{\varepsilon, b, c}, \delta_{1}$-closed to $X_{\varepsilon, b, c}$, such that $\widetilde{X_{\varepsilon, b, c}}$ has at least two limit cycles, $\delta_{2}$-closed to $L_{\varepsilon, b, c}$.

We prove the Theorems 1.1 and 1.2 in sections 5 and 6 . In section 4 we prove that the parameter values for which $X_{\varepsilon, b, c}$ has limit cycles approache $\{\varepsilon=b=0\}$, for $\varepsilon \downarrow 0$.

\section{The Liénard Transformation}

Let $X_{\mu, \nu_{0}, \nu_{1}}$ given by (8). We start with the transformation

$$
\begin{array}{lll}
x=t^{2} x_{1} & \nu_{0}=t^{6} \nu_{0}^{\prime} & \mu=-t^{4} \\
y=t^{3} y_{1} & \nu_{1}=t^{4} \nu_{1}^{\prime} & T=t^{5} .
\end{array}
$$

Divide by $t$ to get

$$
y_{1} \frac{\partial}{\partial x_{1}}+\left[x_{1}^{2}-1+T y_{1}\left(\nu_{0}^{\prime}+\nu_{1}^{\prime} x_{1}+x_{1}^{3}\right)\right] \frac{\partial}{\partial y_{1}} .
$$

Next, use the Liénard transformation

$$
\left\{\begin{array}{l}
T y_{2}=\int T\left(\nu_{0}^{\prime}+\nu_{1}^{\prime} x_{1}+x_{1}^{3}\right) d x_{1}-y_{1} \\
x_{2}=x_{1}
\end{array}\right.
$$


Thus we have

$$
\left[T\left(\nu_{0}^{\prime} x_{2}+\frac{\nu_{1}^{\prime}}{2} x_{2}+\frac{x_{2}^{4}}{4}\right)-T y_{2}\right] \frac{\partial}{\partial x_{2}}-\frac{1}{T}\left(x_{2}^{2}-1\right) \frac{\partial}{\partial y_{2}} .
$$

The Liénard transformation (15) is a diffeomorphism from $\left(x_{1}, y_{1}\right)$ plane to $\left(x_{2}, y_{2}\right)$-plane.

Multiply (16) by $\frac{-1}{T}$ and denote $\varepsilon^{\prime}=\frac{1}{T^{2}}$

$$
\left[y_{2}-\frac{1}{4}\left(4 \nu_{0}^{\prime}+2 \nu_{1}^{\prime} x_{2}^{2}+x_{2}^{4}\right)\right] \frac{\partial}{\partial x_{2}}+\varepsilon^{\prime}\left(x_{2}^{2}-1\right) \frac{\partial}{\partial y_{2}} .
$$

Multiply by 4 and put $y_{3}=4 y_{2}, \nu_{0}^{\prime \prime}=4 \nu_{0}^{\prime}, \nu_{1}^{\prime \prime}=2 \nu_{1}^{\prime}, \varepsilon=\frac{1}{16} \varepsilon^{\prime}$

$$
\left[y_{3}-\left(\nu_{0}^{\prime \prime} x_{3}+\nu_{1}^{\prime \prime} x_{3}^{2}+x_{3}^{4}\right)\right] \frac{\partial}{\partial x_{3}}+\varepsilon\left(x_{3}^{2}-1\right) \frac{\partial}{\partial y_{3}} .
$$

To simplify the desingularization, make the change

$$
\begin{array}{ll}
\widetilde{x}=x_{3}+1 & b=\nu_{0}^{\prime \prime}-2 \nu_{1}^{\prime \prime} \\
\widetilde{y}=y_{3}-1+\nu_{0}^{\prime \prime}-\nu_{1}^{\prime \prime} & c=\nu_{1}^{\prime \prime}-6
\end{array}
$$

and denote $x$ and $y$ again to get (9).

Proposition 2.1. $F_{b, c}: R \rightarrow R$ given by (10) satisfies:

a) If $b=0$ then $F_{b, c}$ has one zero for $c>4,2$ zeroes for $c=4$ or $c=0$ and 3 zeroes for $c<0$ or $0<c<4$.

b) The equation

$$
\left(\frac{3 c-16}{9}\right)^{3}+\left(\frac{-36 c+128-27 b}{54}\right)^{2}=0
$$

defines a cusp on the $(b, c)$-plane which is tangent to $\{b=0\}$ at $(b, c)=(0,0)$, crosses $\{b=0\}$ at $(b, c)=(0,4)$ and crosses $\{c=0\}$ at $(b, c)=(9.48, \ldots, 0)$. The cusp and $\{b=0\}$ divide the $(b, c)$-plane defining the number of zeroes of $F_{b, c}$.

Proof: The equation (20) is $D=0$ with $D$ the discriminant of $\frac{F_{b, c}(x)}{x}=0$ 
Proposition 2.2. $F_{b, c}: R \rightarrow R$ given by (10) satisfies:

a) If $b=0$ then $F_{b, c}$ has 3 critical points for $c<0$ or $0<c<\frac{9}{2}$, 1 critical point for $c>\frac{9}{2}$ and 2 critical points for $c=0$ or $c=\frac{9}{2}$.

b) The equation

$$
\left(\frac{c-6}{6}\right)^{3}+\left(\frac{-2 c-b+8}{8}\right)^{2}=0
$$

defines a cusp on the $(b, c)$-plane which is tangent to $\{b=0\}$ at $(0,0)$, crosses $\{b=0\}$ at $\left(0, \frac{9}{2}\right)$ and $\{c=0\}$ at $(16,0)$. The vertex is the point $(-4,6)$. The cusp and $\{b=0\}$ divide the $(b, c)$-plane defining the number of critical points of $F_{b, c}$.

c) If $c \geq 6$ then $F_{b, c}$ does not have inflexion points and an inflexion point is a critical point if and only if $(b, c)$ satisfies (21).

Proof: The equation (21) is $D=0$ with $D$ the discriminant of $F_{b, c}^{\prime}(x)=0$.

Figure 2 shows two sets of bifurcation: one defined by the equality of critical points and other by the equality of zeroes.

\section{The Singularities of (9)}

If $\varepsilon \neq 0$, the singularities of the vector field $X_{\varepsilon, b, c}$, given by (9), are the points $(0,0)$ and $\left(2, F_{b, c}(2)\right)$. The representative matrix of $D X_{\varepsilon, b, c}$ at $(0,0)$ is given by

$$
\left[\begin{array}{cc}
-b & 1 \\
-2 \varepsilon & 0
\end{array}\right]
$$

The equation of the eigenvalues is

$$
z^{2}+b z+2 \varepsilon=0
$$

If $b^{2}-8 \varepsilon<0$ then $X_{\varepsilon, b, c}$ has a repelling focus for $b<0$ and an attracting focus for $b>0$. The eigenvalues have real part equal zero if and only if $b=0$. We have an attracting node if $b^{2}-8 \varepsilon \geq 0$ and $b>0$, and a repelling node if $b^{2}-8 \varepsilon \geq 0$ and $b<0$.

The representative matrix of $D X_{\varepsilon, b, c}$ at $(2,2 b+4 c-16)$ is given by

$$
\left[\begin{array}{cc}
-(b+4 c-16) & 1 \\
2 \varepsilon & 0
\end{array}\right] .
$$

The equation of the eigenvalues is

$$
z^{2}+(b+4 c-16) z-2 \varepsilon=0
$$

For $\varepsilon>0$ the singularity $(2,2 b+4 c-16)$ is a saddle. The saddle is a critical point of $F_{b, c}$ for the parameter values in the plane given by (12). 
Theorem 3.1. Let $X_{\varepsilon, b, c}$ given by (9). We have:

a) $(x, y)=(0,0)$ is an attracting focus (node) if $b>0$ and a repelling focus (node) if $b<0$.

b) $(x, y)=(0,0)$ is a weakly attracting focus if $b=0$ and $c \geq 12$ and $a$ weakly repelling focus if $b=0$ and $c<12$.

c) For any parameter value in $H=\{(\varepsilon, 0, c) \mid c \neq 12, \varepsilon>0\}$ there exists a Hopf bifurcation of codimension 1 . There exists one attracting limit cycle for the parameter values inside the region $\{(\varepsilon, b, c) \mid \varepsilon>0, b<0, c>12\}$ and there exists one repelling limit cycle for the parameter values inside the region $\{(\varepsilon, b, c) \mid$ $\varepsilon>0, b>0, c<12\}$.

d) For any parameter value in $H_{2}=\{(\varepsilon, 0, c) \mid \varepsilon>0\}$ there exists a Hopf bifurcation of codimension 2. There are two limit cycles for the parameter values inside the region $\{(\varepsilon, b, c) \mid \varepsilon>0, b>0, c<$ $12\}$. The inner one is repelling and the other is attracting.

Proof: The divergence of $X_{\varepsilon, b, c}$ is zero at $(0,0)$ if and only if $b=0$. Taking $b=0, X_{\varepsilon, b, c}$ becomes

$$
\left(y-c x^{2}+4 x^{3}-x^{4}\right) \frac{\partial}{\partial x}+\varepsilon\left(x^{2}-2 x\right) \frac{\partial}{\partial y} .
$$

Make the change

$$
y=\sqrt{2 \varepsilon} y_{1}
$$

and denote $y=y_{1}$ to get

$$
\left(\sqrt{2 \varepsilon} y-c x^{2}+4 x^{3}-x^{4}\right) \frac{\partial}{\partial x}+\left(-\sqrt{2 \varepsilon} x+\frac{\sqrt{2 \varepsilon}}{2} x^{2}\right) \frac{\partial}{\partial y}
$$

Make the change

$$
x=r \cos \theta \quad y=r \sin \theta
$$

to get, in polar coordinates,

$$
P(r, \theta) \frac{\partial}{\partial r}+Q(r, \theta) \frac{\partial}{\partial \theta}
$$


with

$$
P(r, \theta)=\left(\frac{\sqrt{2 \varepsilon}}{2} \sin \theta-c \cos \theta\right) \cos ^{2} \theta r^{2}+4 \cos ^{4} \theta r^{3}-\cos ^{5} \theta r
$$

$$
\begin{aligned}
Q(r, \theta)= & -\sqrt{2 \varepsilon}+\left(\frac{\sqrt{2 \varepsilon}}{2} \cos \theta+c \sin \theta\right) \cos ^{2} \theta r \\
& -4 \cos ^{3} \theta \sin \theta r^{2}+\cos ^{4} \theta \sin \theta r^{3} .
\end{aligned}
$$

The phase portraits of (30) are the graphics of $r=f_{\rho}(\theta), f_{\rho}(0)=\rho$, solutions of

$$
R(r, \theta)=\frac{d r}{d \theta}=R_{1}(\theta) r+R_{2}(\theta) r^{2}+\cdots
$$

with

$$
R_{k}(\theta)=\left.\frac{1}{k !} \frac{\partial^{k} R(r, \theta)}{\partial r^{k}}\right|_{r=0} .
$$

We have

$$
f_{\rho}(\theta)=u_{1}(\theta) \rho+u_{2}(\theta) \rho^{2}+\cdots
$$

For $r=f_{\rho}(\theta)$ the equation (32) becomes

(35) $u_{1}^{\prime}(\theta) \rho+u_{2}^{\prime}(\theta) \rho^{2}+\cdots$

$$
=R_{1}(\theta)\left[u_{1}(\theta) \rho+\cdots\right]+R_{2}(\theta)\left[u_{1}(\theta) \rho+\cdots\right]^{2}+\cdots
$$

Thus, according [ALGM], we get

$$
\begin{aligned}
& u_{1}(\theta)=1 \\
& u_{2}(\theta)=\int R_{2} d s \\
& u_{3}(\theta)=\int\left(2 R_{2} u_{2}+R_{3}\right) d s \\
& u_{4}(\theta)=\int\left[2 R_{2} u_{3}+R_{2} u_{2}^{2}+3 R_{3} u_{2}+R_{4}\right] d s \\
& u_{5}(\theta)=\int\left[2 R_{2}\left(u_{4}+u_{2} u_{3}\right)+3 R_{3}\left(u_{3}+u_{2}^{2}\right)+4 R_{4} u_{2}\right] d s .
\end{aligned}
$$


Define the returning map $\pi: R^{+} \rightarrow R$ by

$$
\pi(\rho)=f_{\rho}(2 \pi)-\rho=u_{2}(2 \pi) \rho^{2}+\cdots
$$

The Lyapunov coefficients are given by

$$
V_{k}=\frac{\pi^{(k)}(0)}{k !} .
$$

The computation, using MAPLE, gives

$$
\begin{aligned}
V_{1}= & 0 \\
V_{2}= & 0 \\
V_{3}= & \frac{\pi(c-12)}{4 \sqrt{2 \varepsilon}} \\
V_{4}= & 0 \\
V_{5}= & p(\varepsilon)\left(-168 c^{2}+21 \varepsilon c-1152 \sqrt{2 \varepsilon} \pi c\right. \\
& \left.+6912 \sqrt{2 \varepsilon} \pi-76 \varepsilon+14 c^{3}+48 \sqrt{2 \varepsilon} \pi c^{2}\right) \\
p(\varepsilon)= & \frac{\sqrt{2} \pi}{1024(\sqrt{2 \varepsilon})^{3}} .
\end{aligned}
$$

When $b=0$ we have $V_{1}=V_{2}=0$ and $V_{3}=0$ if and only if $c=12$. Besides $V_{3}>0$ for $c>12$ and $V_{3}<0$ for $c<12$. Thus $X_{\varepsilon, b, c}$ has a weakly attracting focus for $c>12$ and a weakly repelling focus for $c<12$. For $c=12$ we have

$$
V_{5}=\frac{11}{32} \frac{\pi}{\sqrt{2 \varepsilon}} .
$$

Thus, $(0,0)$ is a weakly attracting focus.

\section{The Phase Portrait of $X_{\varepsilon, b, c}$}

First we are going to study the phase portrait of $X_{\varepsilon, b, c}$ given by (9) when $\varepsilon=0$. In this case we have

$$
\left(y-F_{b, c}(x)\right) \frac{\partial}{\partial x}
$$


For $b$ and $c$ fixed the singularities of (41) are the points $(x, y)$ such that $y=F_{b, c}(x)$. Except for the the critical points, all the points are normally hyperbolic singular points. Figure 3 shows the phase portrait for some values of $(b, c)$.

For $\varepsilon \neq 0$, when $x=0$ and $x=2$ cross transversally the graphic of $y=F_{b, c}(x)$ in non-critical points we can use the R. Lutz and M. Goze lemma $([\mathbf{L G}])$ to sketch the phase portrait of $X_{\varepsilon, b, c}$. The trajectories are so that

$$
\frac{d y}{d x}=\frac{\varepsilon\left(x^{2}-2 x\right)}{y-F_{b, c}(x)} .
$$

Thus the vector field is almost horizontal for $\varepsilon \downarrow 0$.

The main difficulty to obtain the whole phase portrait is the number of limit cycles.

Lemma 4.1 (Copell). Let the vector field given by

$$
(F(x)-y) \frac{\partial}{\partial x}+g(x) \frac{\partial}{\partial y}
$$

satisfying:

i) $F \in C^{2}, g \in C^{1}$ are defined for $x \in(\alpha, \beta)$;

ii) $f(x)=F^{\prime}(x)$ has only one zero $x_{0}<0, f(x)<0(>0)$ when $\alpha<x<x_{0}\left(x_{0}<x<\beta\right)$;

iii) $F(0)=0, F\left(\xi_{0}\right)=0$ with $\alpha<\xi_{0}<x_{0}$;

iv) $x g(x)>0$ if $x \neq 0, x \in(\alpha, \beta)$.

Then there is no limit cycle in $\xi_{0}<x<\beta$.

The proof of the Copell's Lemma uses a symmetry in the line where the divergence is zero $([\mathbf{D R 2}])$.

Consider $b \neq 0$ and $c \in R$ and suppose that $X_{\varepsilon, b, c}$ has a limit cycle $\Gamma_{\varepsilon}$, then either the limit cycle disappear or the limit periodic set will be $(0,0)$ for $\varepsilon \downarrow 0$. In fact, if $b \neq 0$ the singularity $(0,0)$ is not a critical point.

Theorem 4.2. Let $X_{\varepsilon, b, c}$ given by (9) with $b \neq 0$. There exists $\varepsilon_{0}>0$, $\varepsilon_{0}=\varepsilon_{0}(b, c)$, such that if $0<\varepsilon<\varepsilon_{0}$ then $X_{\varepsilon, b, c}$ has no limit cycle. 
Proof: We suppose that for $b, c \in R, b \neq 0$, there exists $\varepsilon_{n} \rightarrow 0$ such that $X_{\varepsilon_{n}, b, c}$ has a limit cycle $\Gamma_{\varepsilon_{n}}$. By the previous remark we have that $\Gamma_{\varepsilon_{n}} \rightarrow\{(0,0)\}$. If $b>0$ then $-X_{\varepsilon_{n}, b, c}$ satisfies (ii), (iii) and (iv) in Lemma 4.1 , for a suitable choose of $(\alpha, \beta)$. Thus $X_{\varepsilon_{n}, b, c}$ has no limit cycle in a neighbourhood of $(0,0)$.

If $b<0$ then we consider the change $x_{1}=-x, y_{1}=y$. The vector field (9) becomes

$$
Y_{\varepsilon_{n}, b, c}=\left(\widetilde{F}_{b, c}\left(x_{1}\right)-y_{1}\right) \frac{\partial}{\partial x_{1}}+\varepsilon\left(x_{1}^{2}+2 x_{1}\right) \frac{\partial}{\partial y_{1}}
$$

The vector field (44) satisfies (ii), (iii) and (iv) and then it has no limit cycle in a neighbourhood of $(0,0)$.

\section{The Desingularization of $X_{\varepsilon, b, c}$}

5.1. The Desingularization at $(x, y, \varepsilon, b, c)=(0,0,0,0,12)$. According with the previous paragraph $(\varepsilon, b, c)=(0,0,12)$ is the limit of the points where we have the Hopf bifurcation of codimension 2, for $\varepsilon \downarrow 0$. The point $(x, y)=(0,0)$ is not a normally hyperbolic singular point when $(\varepsilon, b, c)=(0,0,12)$. To simplify the calculation we start with the change

$$
c_{1}=c-12 .
$$

Denote $c=c_{1}$ to get

$$
X_{\varepsilon, b, c}=\left(y-b x-c x^{2}-12 x^{2}+4 x^{3}-x^{4}\right) \frac{\partial}{\partial x}+\varepsilon\left(x^{2}-2 x\right) \frac{\partial}{\partial y} .
$$

Let $K=\left(S^{2} \times D\right) \cup\left(D^{2} \times S^{1}\right)$ with $S^{2}=\left\{(\bar{\varepsilon}, \bar{b}, \bar{c}) \mid \bar{\varepsilon}^{2}+\bar{b}^{2}+\bar{c}^{2}=1\right\}$, $D=\{(\bar{x}, \bar{y}) \mid \bar{x} \in[0,1], \bar{y} \in[0,1]\}, D^{2}=\left\{(\bar{\varepsilon}, \bar{b}, \bar{c}) \mid \bar{\varepsilon}^{2}+\bar{b}^{2}+\bar{c}^{2}<1, \bar{\varepsilon}>\right.$ $0\}$ and $S^{1}=\left\{(\bar{x}, \bar{y}) \mid \bar{x}^{2}+\bar{y}^{2}=1\right\}$. Consider the map $\varphi:[0, T] \times K \rightarrow \mathbb{R}^{5}$, given by

$$
\begin{array}{ll}
x=u \bar{x} & \varepsilon=u^{2} \bar{\varepsilon} \\
y=u^{2} \bar{y} & b=u \bar{b} \\
c=u \bar{c}
\end{array}
$$

with $u \in[0, T],(\bar{x}, \bar{y}, \bar{\varepsilon}, \bar{b}, \bar{c}) \in K$ and $T>0$. 
There exists $\widehat{X}$ on $[0, T] \times K$ such that $\varphi_{*}(\widehat{X})=X_{\varepsilon, b, c}$. Dividing $\widehat{X}$ by $u$ the vector field resulting $\bar{X}$ will be "the desingularized vector field" in $[0, T] \times K$.

(48) $\bar{X}_{u, \bar{b}, \bar{c}}=\left(\bar{y}-\bar{b} \bar{x}-u \overline{c x}^{2}-12 \bar{x}^{2}+4 u \bar{x}^{3}-u^{2} \bar{x}^{4}\right) \frac{\partial}{\partial \bar{x}}+\bar{\varepsilon}\left(u \bar{x}^{2}-2 \bar{x}\right) \frac{\partial}{\partial \bar{y}}$.

Let $M_{0}=\{(x, y)\} \times\{(\varepsilon, b, c)\}$ and $T_{1}=[0, T] \times K$. We define $M_{1}=$ $\left(T_{1} \cup M_{0}-\{0\}\right) /(m \sim \varphi(m))$. Let $\phi: M_{1} \rightarrow M_{0}$ such that $\phi \mid(K \times\{0\})=$ $\varphi$ and $\phi \mid M_{0}-\{0\}=$ id. Consider $\pi: M_{0} \rightarrow\{(\varepsilon, b, c)\}$ given by $\pi(x, y, \varepsilon, b, c)=(\varepsilon, b, c)$ and $\widehat{\pi}: M_{1} \rightarrow\{(\varepsilon, b, c)\}$ by $\widehat{\pi}(x, y, \varepsilon, b, c)=$ $\pi(\phi(x, y, \varepsilon, b, c))$.

Let $\bar{\lambda}_{0}=\left(\overline{\varepsilon_{0}}, \overline{b_{0}}, \overline{c_{0}}\right) \in S^{2}, l_{\bar{\lambda}_{0}}=\left\{\left(u^{2} \overline{\varepsilon_{0}}, u \overline{b_{0}}, u \overline{c_{0}}\right) \mid u>0\right\}$ and $P_{\bar{\lambda}_{0}}=$ $\widehat{\pi}^{-1}\left(l_{\bar{\lambda}_{0}}\right)$. We have that $P_{\bar{\lambda}_{0}}$ is a 3 -dimensional space.
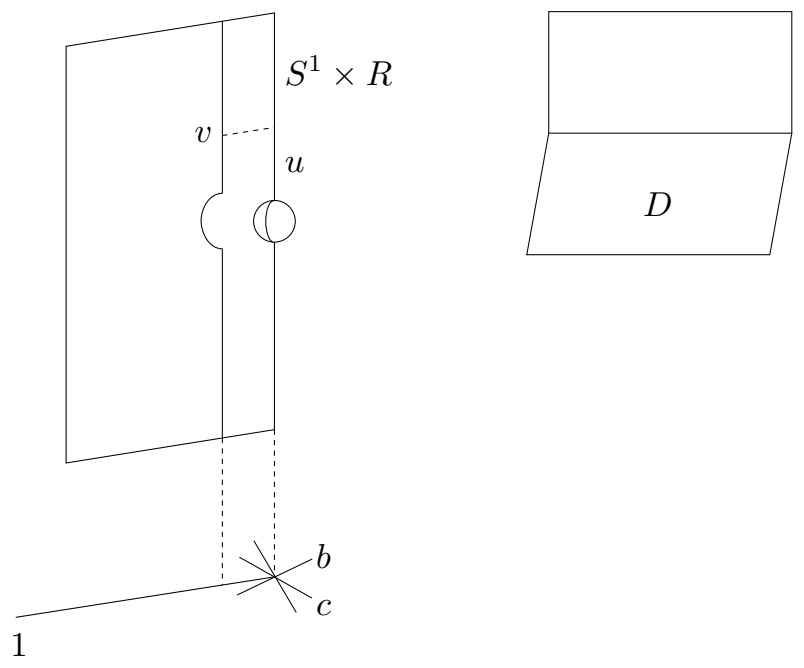

Figure 6. Foliation of $M_{1}$.

Define $\widetilde{X}$ on $M_{1}$ by

$$
\tilde{X}= \begin{cases}X_{\varepsilon, b, c} & \text { on } M_{1}-\{0\} \\ \bar{X} & \text { on } T_{1} .\end{cases}
$$


On $S^{1} \times \mathbb{R}$ we have $\bar{\varepsilon}=\bar{b}=\bar{c}=0$. Make the change

$$
x=u \cos \theta \quad y=u^{2} \sin \theta
$$

and denote $c=\cos \theta$ and $s=\sin \theta$ to get

(51) $\frac{u c}{c^{2}+2 s^{2}}\left(s-12 c^{2}+4 u c^{2}-u^{2} c^{4}\right) \frac{\partial}{\partial u}$

$$
-\frac{2 s}{c^{2}+2 s^{2}}\left(s-12 c^{2}+4 u c^{2}-u^{2} c^{4}\right) \frac{\partial}{\partial \theta} \text {. }
$$

The singularities of $(51)$ are $(0,0),(\pi, 0),\left(\theta_{1}, 0\right)$ and $\left(\theta_{2}, 0\right)$ with $\theta_{1}$ and $\theta_{2}$ solutions of $s=12 c^{2}$. We have $\theta_{1} \in\left(0, \frac{\pi}{2}\right)$ and $\theta_{2} \in\left(\frac{\pi}{2}, \pi\right)$. Multiplying (51) by $\left(c^{2}+2 s^{2}\right)$ we have

$$
\begin{aligned}
& X(\theta, u)=-2 s\left(s-12 c^{2}+4 u c^{2}-u^{2} c^{4}\right) \frac{\partial}{\partial \theta} \\
& +u c\left(s-12 c^{2}+4 u c^{2}-u^{2} c^{4}\right) \frac{\partial}{\partial u} \\
& D X(\theta, 0)=\left[\begin{array}{cc}
-4 s c+24 c^{3}-48 s^{2} c & -8 c^{2} s \\
0 & s c-12 c^{3}
\end{array}\right] \\
& D X(0,0)=\left[\begin{array}{cc}
24 & 0 \\
0 & -12
\end{array}\right] \quad D X(\pi, 0)=\left[\begin{array}{cc}
-24 & 0 \\
0 & 12
\end{array}\right] \text {. }
\end{aligned}
$$

For $(\theta, u)=(0,0)$ the angular eigenvalue is positive and the radial eigenvalue is negative. For $(\theta, u)=(\pi, 0)$ the angular eigenvalue is negative and the radial eigenvalue is positive.

If $\theta=\theta_{1}\left(\theta=\theta_{2}\right)$ the angular eigenvalue $-4 s c+24 c^{3}-48 s^{2} c=$ $-2 s c-48 s^{2} c$ is negative (positive) and the radial eigenvalue is zero. Thus we have

$$
D X\left(\theta_{1}, 0\right)=\left[\begin{array}{cc}
<0 & \neq 0 \\
0 & 0
\end{array}\right] \quad D X\left(\theta_{2}, 0\right)=\left[\begin{array}{cc}
>0 & \neq 0 \\
0 & 0
\end{array}\right]
$$


When $\bar{\varepsilon}=1$ and $u=0$ in (48) we have

$$
\bar{X}_{\bar{b}, \bar{c}}=\left(\bar{y}-\bar{b} \bar{x}-12 \bar{x}^{2}\right) \frac{\partial}{\partial \bar{x}}-2 \bar{x} \frac{\partial}{\partial \bar{y}}
$$
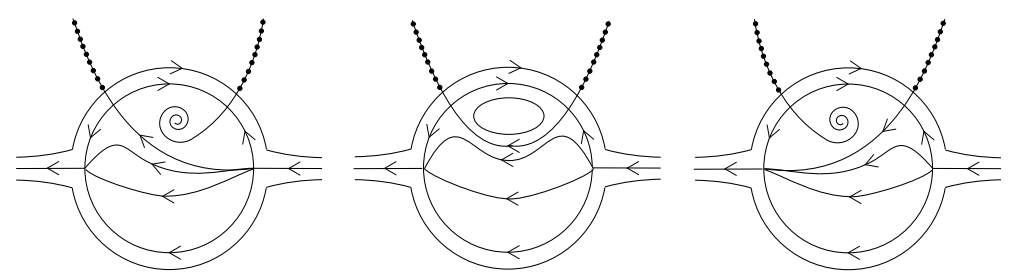

Figure 7. Phase Portraits of $\tilde{X}$ for $\bar{b}>0, \bar{b}=0$ and $\bar{b}<0$.

$\bar{X}_{\bar{b}, \bar{c}}$ independs of $\bar{c}$ and when $\bar{b}=0, \bar{X}_{\bar{b}, \bar{c}}$ has a center in $(\bar{x}, \bar{y})=(0,0)$.

$$
\bar{X}_{0}=\left(\bar{y}-12 \bar{x}^{2}\right) \frac{\partial}{\partial \bar{x}}-2 \bar{x} \frac{\partial}{\partial \bar{y}}
$$

If we take $\bar{\varepsilon}=1, \bar{b}=\bar{b}_{0}>0, u=0$ or $\bar{\varepsilon}=1, \bar{b}=\bar{b}_{1}<0, u=0$ the phase portraits are illustrated in Figure 7 .

5.2. The desingularization at $(x, y, \varepsilon, b, c)=(2,0,0,0,4)$. In this case $F_{0,4}(2)=0$. We start with the change

$$
\begin{array}{ll}
x_{1}=x-2 & b_{1}=-b-4 c+16 \\
y_{1}=y+2 b-4 c+16 & c_{1}=c-4 .
\end{array}
$$

Denote $x, y, b, c$ to get

$$
\left(y+b x-c x^{2}-4 x^{2}-4 x^{3}-x^{4}\right) \frac{\partial}{\partial x}+\varepsilon\left(x^{2}+2 x\right) \frac{\partial}{\partial y} .
$$

Consider the map given by (47) and $\bar{\varepsilon}=1$

$$
\left(\bar{y}+\bar{b} \bar{x}-4 \bar{x}^{2}-4 u \bar{x}^{3}-u^{2} \bar{x}^{4}\right) \frac{\partial}{\partial \bar{x}}+\left(2 \bar{x}+u \bar{x}^{2}\right) \frac{\partial}{\partial \bar{y}} .
$$

If $\bar{b}=\bar{c}=u=0$ then

$$
\left(\bar{y}-4 \bar{x}^{2}\right) \frac{\partial}{\partial \bar{x}}+2 \bar{x} \frac{\partial}{\partial \bar{y}}
$$


has a singular point $(0,0)$ and

$$
D X(0,0)=\left[\begin{array}{ll}
0 & 1 \\
2 & 0
\end{array}\right] .
$$

Thus $(0,0)$ is a saddle point.

Now we use the same steps used in (50), (51), (52), (53) and (54):

$$
\begin{aligned}
& \frac{-2 u s}{c^{2}+2 s^{2}}\left(s-4 c^{2}-4 u c^{3}-u^{2} c^{4}\right) \frac{\partial}{\partial \theta} \\
& +\frac{u^{2} c}{c^{2}+2 s^{2}}\left(s-4 c^{2}-4 u c^{3}-u^{2} c^{4}\right) \frac{\partial}{\partial u} \\
& \text { (63) } \quad-2 s\left(s-4 c^{2}-4 u c^{3}-u^{2} c^{4}\right) \frac{\partial}{\partial \theta} \\
& +u c\left(s-4 c^{2}-4 u c^{3}-u^{2} c^{4}\right) \frac{\partial}{\partial u} \\
& \text { (64) } D X(\theta, u)=\left[\begin{array}{cc}
-4 s c-16 s^{2} c+8 c^{3} & 8 s c^{3} \\
0 & s c-4 c^{3}
\end{array}\right] \\
& \text { (65) } \quad D X(\pi, 0)=\left[\begin{array}{cc}
-8 & 0 \\
0 & 4
\end{array}\right] \quad D X(0,0)=\left[\begin{array}{cc}
8 & 0 \\
0 & -4
\end{array}\right] \\
& \text { (66) } D X\left(\varphi_{1}, 0\right)=\left[\begin{array}{cc}
<0 & \neq 0 \\
0 & 0
\end{array}\right] \quad D X\left(\varphi_{2}, 0\right)=\left[\begin{array}{cc}
>0 & \neq 0 \\
0 & 0
\end{array}\right]
\end{aligned}
$$

for $\varphi_{1}$ and $\varphi_{2}$ solutions of $\sin \varphi=4 \cos \varphi$.

5.3. The desingularization at $(x, y, \varepsilon, b, c)=(1,1,0,0,4)$. We start with the change

$$
\begin{array}{ll}
x_{1}=x-1 & b_{1}=-b-2 c+8 \\
y_{1}=y-b-c+3 & c_{1=} c-4 .
\end{array}
$$

Denote $x, y, b, c$ to get

$$
\left(y+b x-c x^{2}+2 x^{2}-x^{4}\right) \frac{\partial}{\partial x}+\varepsilon\left(x^{2}-1\right) \frac{\partial}{\partial y} .
$$

Consider the map $\delta:[0, T] \times S_{+}^{4} \rightarrow R^{5}$ given by

$$
\begin{array}{ll}
x=u \bar{x} & b=u \bar{b} \\
y=u^{2} \bar{y} & c=u \bar{c} .
\end{array}
$$


Make $\bar{X}=\frac{1}{u} X$ and take $\bar{\varepsilon}=1$

$$
\left(\bar{y}+\bar{b} \bar{x}-u \overline{c x}^{2}+2 \bar{x}^{2}-u^{2} \bar{x}^{4}\right) \frac{\partial}{\partial \bar{x}}+\left(u^{2} \bar{x}^{2}-1\right) \frac{\partial}{\partial \bar{y}} .
$$

For $\bar{b}=\bar{c}=u=0$ we have

$$
\left(\bar{y}+2 \bar{x}^{2}\right) \frac{\partial}{\partial \bar{x}}-\frac{\partial}{\partial \bar{y}}
$$

It has no singular point. Using again the same steps we have

$$
\begin{aligned}
& \frac{-2 s}{\left(c^{2}+2 s^{2}\right)}\left(s+2 c^{2}-u^{2} c^{4}\right) \frac{\partial}{\partial \theta} \\
& \quad+\frac{u c}{\left(c^{2}+2 s^{2}\right)}\left(s+2 c^{2}-u^{2} c^{4}\right) \frac{\partial}{\partial u} \\
& -2 s\left(s+2 c^{2}-u^{2} c^{4}\right) \frac{\partial}{\partial \theta}+u c\left(s+2 c^{2}-u^{2} c^{4}\right) \frac{\partial}{\partial u} \\
& D X(\theta, 0)=\left[\begin{array}{cc}
-4 s c-4 c^{3}+8 c s^{2} & 0 \\
0 & s c+2 c^{3}
\end{array}\right] \\
& D X(0,0)=\left[\begin{array}{cc}
-4 & 0 \\
0 & 2
\end{array}\right] \quad D X(\pi, 0)=\left[\begin{array}{cc}
4 & 0 \\
0 & -2
\end{array}\right] \\
& D X\left(\theta_{1}, 0\right)=\left[\begin{array}{cc}
<0 & 0 \\
0 & 0
\end{array}\right] \quad D X\left(\theta_{2}, 0\right)=\left[\begin{array}{cc}
>0 & 0 \\
0 & 0
\end{array}\right]
\end{aligned}
$$

for $\theta_{1}$ and $\theta_{2}$ solutions of $s+2 c^{2}=0$.
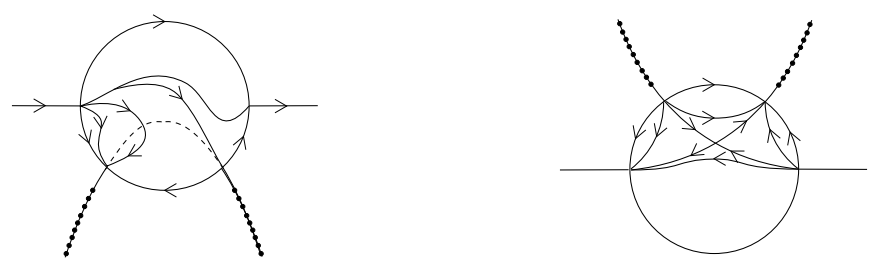

Figure 8 . The Desingularization at $(1,1,0,0,4)$ and at $(2,0,0,0,4)$. 


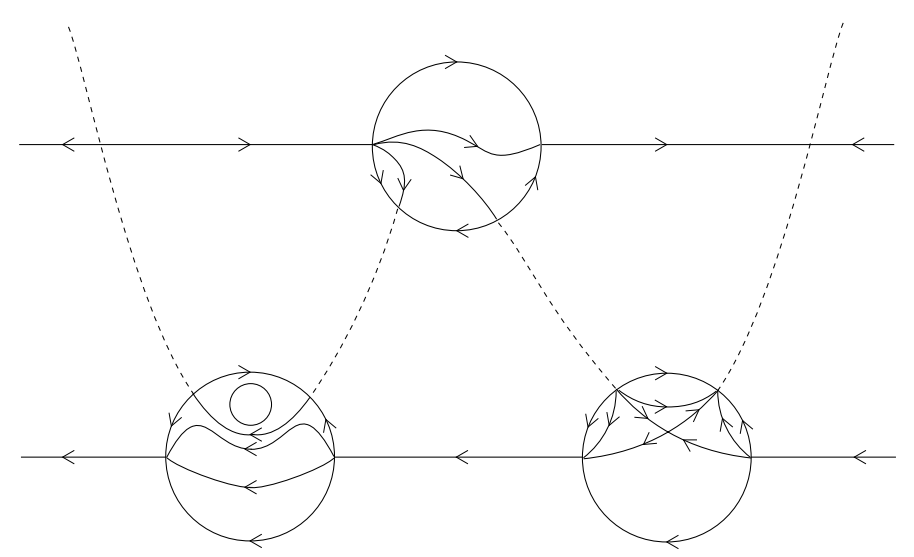

Figure 9. Simultaneous Desingularization.

\section{The Canard Phenomenon}

6.1. Proof of Theorem 1.1. To simplify the calculations we use the change (45). First we prove that there exists a hamiltonian $H$ with integrating factor $K$ such that $K \bar{X}_{0}=d H, \bar{X}_{0}$ given by (56).

Let the change

$$
X=\bar{x} \quad Y=\bar{y}-12 \bar{x}^{2} .
$$

Thus (56) becomes

$$
Y \frac{\partial}{\partial X}+(-2 X-24 X Y) \frac{\partial}{\partial Y} .
$$

Let $F(X, Y)$ given by

$$
F(X, Y)=144 X^{2}+(1+12 Y)-\ln (1+12 Y) .
$$

We have that $F(X, Y)$ is a first integral of (78). In fact

$$
\left(F_{X}, F_{Y}\right)(Y,-2 X-24 X Y)=0 .
$$

Let $G(\bar{x}, \bar{y})$ and $H(\bar{x}, \bar{y})$ given by

$$
\begin{aligned}
& G(\bar{x}, \bar{y})=(1+12 \bar{y})-\ln \left(1+12 \bar{y}-144 \bar{x}^{2}\right) \\
& H(\bar{x}, \bar{y})=\exp (1-12 \bar{y})\left(1+12 \bar{y}-144 \bar{x}^{2}\right) .
\end{aligned}
$$


We have

$$
\begin{aligned}
& H_{\bar{x}}=-(144 \exp (1-12 \bar{y}))(2 \bar{x}) \\
& H_{\bar{y}}=-(144 \exp (1-12 \bar{y}))\left(\bar{y}-12 \bar{x}^{2}\right) .
\end{aligned}
$$

Thus

$$
K(\bar{x}, \bar{y})=-144 \exp (1-12 \bar{y})
$$

is the integrating factor and the hamiltonian $H$ is given by (81).

Now we consider $P_{(1,0,0)}$ defined in section 5 . We have that $P_{(1,0,0)}$ is a 3 -dimensional space and we can look a point in $P_{(1,0,0)}$ with coordinates $(u, v, \theta)$, indicates in the Figure 6 .
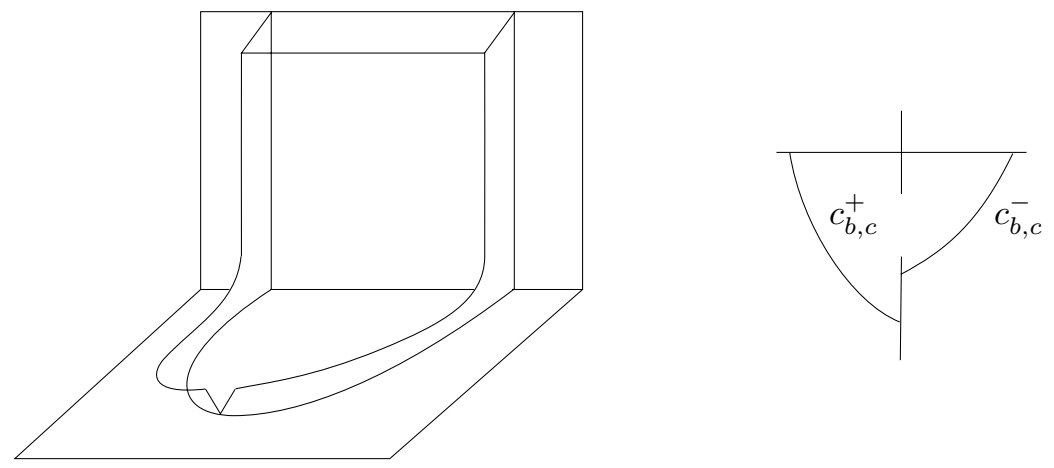

Figure 10. Center Manifold $C_{N}$.

For $u=0$, we denote $D$ the set associated to $v=0$. Let $R$ a rectangle on $P_{(1,0,0)}$ with one side $r$ on $D$ and such that $R$ is transversal to $\partial P$. We take $R$ such that the connection $c$ joing $\left(\theta_{1}, 0\right)$ and $\left(\theta_{2}, 0\right)$ is transversal to $r$ at its middle point. Let $S$ a subrectangle in $R$ with one side $s$ on $D$ and such that $c \cap r$ is the middle of $s$. Let $N=\left\{\left(2, F_{b, c}(2)\right)\right.$ $\left.b, c \in R, 0 \leq \varepsilon \leq \varepsilon_{0}\right\}$ composed by saddle points of $X_{\varepsilon, b, c}$. We take $C_{N}$, the sature of $N$, that is, the closure of the union of segments of orbits of $X_{(u, v, \theta)}$ through the points on $N$ and taken between the first intersection of this trajectory with $S$ in negative time and with $R$ in positive time.

Let $w_{u, \bar{b}, \bar{c}}$ the dual 1-form associated to (48)

$$
\begin{aligned}
w_{u, \bar{b}, \bar{c}}=\left(\bar{y}-\bar{b} \bar{x}-u \overline{c x}^{2}-12 \bar{x}^{2}+4 u \bar{x}^{3}-u^{2} \bar{x}^{4}\right) & d \bar{y} \\
& -\left(u \bar{x}^{2}-2 \bar{x}\right) d \bar{x} .
\end{aligned}
$$


We have

(85)

$$
\begin{aligned}
-144 e^{(1-12 \bar{y})} w_{u, \bar{b}, \bar{c}} & =d H(\bar{x}, \bar{y})-M(\bar{x}, \bar{y}) \\
M(\bar{x}, \bar{y}) & =144 e^{(1-12 \bar{y})}\left[\left(-\bar{b} \bar{x}-u \overline{c x}^{2}+4 u \bar{x}^{3}-u^{2} \bar{x}^{4}\right) d \bar{y}-u \bar{x}^{2} d \bar{x}\right] .
\end{aligned}
$$

Consider the section $[\alpha, \beta]$ joing the center $\alpha=(0,0)$ and $\beta=\left(0,-\frac{1}{12}\right)$ in $c$. We parametrize $[\alpha, \beta]$ by the value of the hamiltonian $H$. Let $C_{h}=H^{-1}(h)$ for $h \in(0, e)\left(H(0,0)=e, H\left(0,-\frac{1}{12}\right)=0\right)$.

The assymptotic development of $P_{u, \bar{b}, \bar{c}}$ (Poincaré Mapping), using the Perturbation Lemma ([DRS1]), is given by

$$
P_{u, \bar{b}, \bar{c}}(h)=h+\bar{b} I_{1}(h)+\bar{c} I_{2}(h)+u I_{3}(h)+o(|u, \bar{b}, \bar{c}|)
$$

with

$$
\begin{aligned}
& I_{1}(h)=144 \int_{C_{h}} e^{(1-12 \bar{y})} \bar{x} d \bar{y} \\
& I_{2}(h)=144 u \int_{C_{h}} e^{(1-12 \bar{y})} \bar{x}^{2} d \bar{y}=0 \\
& I_{3}(h)=-576 \int_{C_{h}} e^{(1-12 \bar{y})} \bar{x}^{3} d \bar{y}+144 \int_{C_{h}} e^{(1-12 \bar{y})} \bar{x}^{2} d \bar{x} .
\end{aligned}
$$

If $u=h=0$ the development of the separation between $C_{b, c}^{+}$and $C_{b, c}^{-}$ is given by

$$
\Delta(\bar{b}, \bar{c})=\bar{b} I_{1}(0) .
$$

If $\{h=f(u, \bar{b}, \bar{c})\}$ and $\{h=g(u, \bar{b}, \bar{c})\}$ represent the intersections of $C_{N}(\bar{b}, \bar{c})$ with $R$ then we can extend (88)

$$
f(u, \bar{b}, \bar{c})-g(u, \bar{b}, \bar{c})=\bar{b} I_{1}(0)+u I_{3}(0)+o(|u, \bar{b}, \bar{c}|) .
$$

Let $\Delta(u, b, c)$ given by

$$
\Delta(u, b, c)=f(u, \bar{b}, \bar{c})-g(u, \bar{b}, \bar{c}) .
$$

For $h=0$ we have $K(\bar{x}, \bar{y})=-144 e^{\left(2-144 x^{2}\right)}$ and $d \bar{y}=24 \bar{x} d \bar{x}$. By integrating by parts we have $I_{1}(0) \neq 0, I_{2}(0)=0$ and $I_{3}(0)=0$. Thus the equations

$$
\Delta=0 \quad \frac{\partial \Delta}{\partial \bar{b}} \neq 0
$$

define $S=\{\bar{b}=\varphi(\bar{c}, u)\}$, the Canard Surface. 
The homoclinic orbits $\gamma$ associated to the parameter values on $S$ are attracting because the parameter is near of $(0,0,12)$ (see $(12))$. In order to study the stability of the limit cycles $\gamma$ associated to the parameter values on $S_{32}$ we must to compute $\int_{\gamma} \operatorname{div} X_{\varepsilon, b, c}$.

We aproach the integral

$$
\text { 92) } \begin{aligned}
& \int_{\gamma} \operatorname{div} X_{\varepsilon, b, c} \\
\simeq & \int_{-1.3013}^{2}\left(b+2 c x-12 x^{2}+4 x^{3}\right)\left(\sqrt{1+\left(24 x-12 x^{2}+4 x^{3}\right)^{2}}+1\right) d x .
\end{aligned}
$$

The computation, using MAPLE, gives

$$
\begin{aligned}
& S_{1}=\operatorname{subs}(b=0.0001, c=12.0001) \simeq-518.12 \\
& S_{2}=\operatorname{subs}(b=0.0001, c=11.9999) \simeq-523.55 \\
& S_{3}=\operatorname{subs}(b=-0.0001, c=11.9999) \simeq-537.06 \\
& S_{4}=\operatorname{subs}(b=-0.0001, c=12.0001) \simeq-531.63 .
\end{aligned}
$$

The values given by (93) attest that the limit cycles are attracting for the parameter values $(\varepsilon, b, c) \in S_{32}$.

Remark 6.1. The "Canard phenomenon" consists in a rapid variation of the shape of the periodic orbits in function of the variation of the parameter. According Theorems 1.1 and 3.1 one can find $X_{\varepsilon_{1}, b_{1}, c_{1}}$ and $X_{\varepsilon_{2}, b_{2}, c_{2}}$ two perturbations of $X_{0,0,12}$ such that:

a) The phase portrait of $X_{\varepsilon_{1}, b_{1}, c_{1}}$ has two limit cycles contained in a small neighbourhood of $(0,0)$, the inner one is repelling and the other is attracting.

b) The phase portrait of $X_{\varepsilon_{2}, b_{2}, c_{2}}$ has an attracting limit cycle contained in a small neighbourhood of $\Gamma_{0,12}^{32}$ (see Figure 5).

Proof of Theorem 1.2. The surface $L$ is obtained implicitly of the same way that canard surface. To make it we consider $\gamma=$ $\left\{\left(2, F_{b, c}(2)\right) \mid b, c \in R, 0 \leq \varepsilon \leq \varepsilon_{0}\right\}$ composed by saddle points of $X_{\varepsilon, b, c}$. Saturing $\gamma$ by the flow of $X_{\varepsilon, b, c}$ we have that the intersections of the manifolds define the homoclinic bifurcation surface.

Let $l(\varepsilon, b, c)$, the homoclinic loop associated to the parameters $(\varepsilon, b, c)$. There is not limit of $l(\varepsilon, b, c)$, for $\varepsilon \downarrow 0$ and $c<4$, for the Hausdorff distance. In fact, the unstable separatrice does not touch the slow manifold for $\varepsilon \downarrow 0$. 


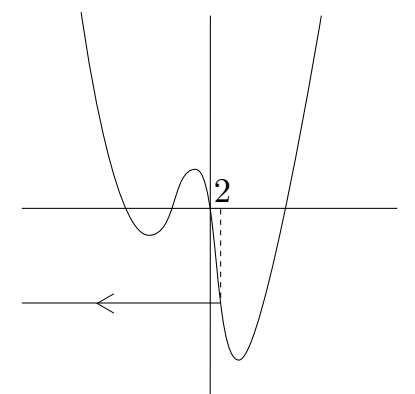

Figure 11. Non Existence of Limit Homoclinic Loops in $\{b=0, c<4\}$.

The parameter values for which $X_{\varepsilon, b, c}$ has limit cycles aproache $\{b=0\}$ for $\varepsilon \downarrow 0$. Thus, the surface $L$ aproaches $\{b=0\}$, for $\varepsilon \downarrow 0$, and the parameter values for which $X_{\varepsilon, b, c}$ has non-generic homoclinic loops aproach $(\varepsilon, b, c)=(0,0,4)$, for $\varepsilon \downarrow 0$.

We need to precise the codimension of the non-generic homoclinic loops. We start with the desingularization of $X_{\varepsilon, b, c}$ in $(x, y, \varepsilon, b, c)=$ $(0,0,0,0,4)$. Let the change

$$
c_{1}=c-4 .
$$

Denote $c$ and make the change (47) to get

$$
\left(\bar{y}-\bar{b} \bar{x}-4 \bar{x}^{2}-u \overline{c x}^{2}+4 u \bar{x}^{3}-u^{2} \bar{x}^{4}\right) \frac{\partial}{\partial \bar{x}}+\left(u \bar{x}^{2}-2 \bar{x}\right) \frac{\partial}{\partial \bar{y}} .
$$

$H(\bar{x}, \bar{y})$ and $K(\bar{x}, \bar{y})$ given by

(96) $H(\bar{x}, \bar{y})=(\exp (1-4 \bar{y}))\left(1+4 \bar{y}-16 \bar{x}^{2}\right) \quad K(\bar{x}, \bar{y})=16 \exp (1-4 \bar{y})$

satisfy

$$
K X_{0}=-H_{\bar{y}} \frac{\partial}{\partial \bar{x}}+H_{\bar{x}} \frac{\partial}{\partial \bar{y}} .
$$

Let the map

$$
R(h)=\bar{b} I_{1}(h)+u \bar{c} I_{2}(h)+u I_{3}(h)+o(u, \bar{b}, \bar{c})
$$

with $I_{1}, I_{2}$ and $I_{3}$ given by

$$
\begin{aligned}
& I_{1}(h)=-\int_{c_{h}} K \bar{x} d \bar{y} \\
& I_{2}(h)=-\int_{c_{h}} K \bar{x}^{2} d \bar{y}=0 \\
& I_{3}(h)=\int_{c_{h}} 4 K \bar{x}^{3} d \bar{y}-\int_{c_{h}} K \bar{x}^{2} d \bar{x} .
\end{aligned}
$$


The equation $R(h)=0$ defines the surface $L$ and besides $R^{\prime}(h)$ is finite if and only if the divergence at the saddle point is zero $[\mathbf{R}]$. Thus $R^{\prime}(h)$ is finite if and only if $\bar{b}=-4 \bar{c}$. To prove that the homoclinic loop is of codimension 2 we need to prove that $R^{\prime}(h) \neq 0$. We use the map $\widetilde{R}(h)=\bar{b}+u \frac{I_{3}(h)}{I_{1}(h)}$ and we have

$$
\widetilde{R}^{\prime}(h)=u\left(\frac{I_{3}(h)}{I_{1}(h)}\right)^{\prime} .
$$

With a similar argument used in $[\mathbf{C W}]$, one can prove that

$$
\left(\frac{I_{3}(h)}{I_{1}(h)}\right)^{\prime}<0
$$

for $h<e$. It follows that $\widetilde{R}^{\prime}(h)<0$.

\section{References}

[ALGM] A. Andronov, E. Leontovich, I. Gordon And A. Maier, "Theory of Bifurcations of Dynamical Sytems on a Plane," I.P.S.T, Jerusalem, 1971.

[CW] L. Chow AND L. WANG, Uniqueness of periodic orbits in some vector fields with cod. 2 singularities, J. Differential Equations 77(2) (1989), 231-253.

[D] F. Dumortier, Local study of planar vector fields: singularities and their unfoldings, Stud. Math. Phys. 2 (1991), 161-241.

[DR1] F. Dumortier and R. Roussarie, Canard cycles and center manifolds, Mem. Amer. Math. Soc. 121(577) (1996), 1-101.

[DR2] F. Dumortier and C. Rousseau, Cubic Liénard equations with linear damping, Nonlinearity 3 (1990), 1015-1039.

[DRS1] F. Dumortier, R. Roussarie and J. Sotomayor, Generic 3-parameter families of vector fields on the plane, unfolding a singularity with nilpotent linear part-The cusp case, Ergodic Theory Dynam. Systems 7 (1987), 375-413.

[DRS2] F. Dumortier, R. Roussarie and J. Sotomayor, Generic 3-parameter families of planar vector fields, unfoldings of saddle, focus and elliptic singularities with nilpotent linear part, in "Bifurcations of Planar Vector Fields: Nilpotent Singularities and Abelian Integrals," (F. Dumortier et al., eds.), Lect. Notes in Math. 1480, Springer Verlag, Berlin, Heidelberg, 1991, pp. 1-164. 
[E] W. EckHAus, Relaxation oscilations including a standard chase on French ducks, in "Asymptotic Analysis II," Lect. Notes in Math. 985, Springer Verlag, Berlin, New York, 1983, pp. 449-494.

[LG] R. Lutz And M. Goze, "Non Standard Analysis. A Pratical Guide with Applications," Lect. Notes in Math. 881, Springer Verlag, Berlin, New York, 1981.

[M] P. MARDEsic, Deploiment versel du cusp d'ordre $n$, These, Université de Bourgogne (1992).

[R] R. Roussarie, On the number of limit cycles which appear by perturbation of a separatix loop of planar vector fields, Bol. Soc. Brasil. Mat. 17 (1986), 67-101.

[RW] R. Roussarie And F. Wagener, A study of the BogdanovTakens bifurcation, Resenhas 2(1) (1995), 1-25.

\author{
Departamento de Matemática-IBILCE-UNESP \\ Rua Cristóvão Colombo 2265 \\ CEP 15054-000 \\ São José do Rio Preto - São Paulo \\ BRAZIL \\ e-mail: prs@mat.ibilce.unesp.br
}

Primera versió rebuda el 16 de març de 1998 , darrera versió rebuda el 26 d'octubre de 1998 